\title{
Der wissenschaftliche Artikel in der germanistischen Linguistik als Modell für den Unterricht wissenschaftlichen Schreibens: Ergebnisse einer Pilotstudie
}

\author{
Birgit Huemer (Luxemburg)
}

\begin{abstract}
Similar structures in research articles of different disciplines indicate that the research article can be qualified as a genre across disciplinary borders. In spite of these structural similarities, which are constitutive for a genre, there are linguistic varieties that can be found in research articles of several disciplines relevant to be pointed out when teaching academic writing at the university. Little research has been conducted so far on linguistic varieties within subcategories of a discipline, because it is assumed that linguistic varieties within the genre research article are discipline-specific and therefore can only be found across disciplines.

This article will present the results of a pilot study in which linguistic varieties within the discipline German linguistics have been researched. 14 introductions to research articles within this field have been selected in order to analyse their macro structure in detail. The data has been taken from a high rated journal in the field of German linguistics called Zeitschrift für germanistische Linguistik (ZGL 2014) in which the most important articles that have been published in the year before are promoted.

The study shows that the macro structure of the research articles' introductions are essentially determined by the research question and the research method, which vary within this discipline. This result is particularly relevant for the teaching of academic writing, because it offers an alternative approach to support the writing process oriented towards the core of every research: which is to formulate a research question and to choose an appropriate method rather than to strictly follow assumed disciplinary writing norms.
\end{abstract}

\section{$1 \quad$ Einleitung}

Seit den 70er Jahren des 20. Jahrhunderts beschäftigt sich die anglo-amerikanische linguistische Forschung mit dem wissenschaftlichen Artikel. Dabei können zwei Forschungsbereiche festgemacht werden, die sich zum Teil ergänzen. Einerseits die Textsorten- oder Genreforschung und andererseits die stark anwendungsorientierte Erforschung professioneller und studentischer Textproduktion (vgl. Bazerman 1988; Biber et al. 2002; Halliday/Martin 1993; Hyland 1998, 2009; Jones/Turner/Street 1999; Lea 1999; Swales 1990, 2004). 
Innerhalb der deutschsprachigen Forschungslandschaft gilt Ehlich (1995) als einer der Hauptvertreter linguistischer Forschung im Bereich der Wissenschaftskommunikation. Seine Ansätze haben ähnlich der anglo-amerikanischen Forschung auch im deutschen Sprachraum konkrete schreibdidaktische Anwendungen inspiriert (vgl. Ehlich/Graefen 2001; Ehlich/Steets 2003). Eine umfassende Darstellung der mittlerweile recht beträchtlichen Anzahl an internationalen Studien und Ansätzen im Bereich wissenschaftlicher Textsorten- und Schreibforschung lässt sich in diesem Artikel nicht bewerkstelligen. Für einen guten Überblick über die wichtigsten anglo-amerikanischen und deutschsprachigen Ansätze im Bereich der text- und diskursanalytischen Forschung zum wissenschaftlichen Schreiben sei daher auf Gruber (2011) verwiesen.

Aufbauend auf die hier erwähnten Forschungsergebnisse und Theorien im Bereich Textsorten und wissenschaftliches Schreiben beschäftigt sich dieser Artikel mit der Frage der disziplinspezifischen Varianten innerhalb der Textsorte wissenschaftlicher Artikel im deutschen Sprachraum. Untersuchungen zu disziplinspezifischen Gemeinsamkeiten und Unterschieden des deutschsprachigen wissenschaftlichen Artikels gibt es nur wenige (vgl. Adamzik/Antos/Jakobs 1997; Busch-Lauer 1997, 2001; Danneberg 1993; Göpferich 1995). Graefen (1997: 9) vermeint im Detail Unterschiede zwischen den Disziplinen festzustellen, spricht aber von einem Konzept des wissenschaftlichen Artikels, da diese Texte durch die ihnen allen zugrunde liegende wissenschaftliche Praxis sozial und sprachlich einheitlich bestimmbar sind. Gray (2011) kommt in ihrer korpuslinguistischen Studie ebenfalls zu dem Schluss, dass man in unterschiedlichen Disziplinen linguistische Varianten findet. Zudem behauptet sie aber, dass man bei diesen Varianten durchaus von verschiedenen Texttypen sprechen kann. In beiden Untersuchungen werden disziplinspezifische Varianten des wissenschaftlichen Artikels festgestellt. Offen bleibt allerdings die Frage, ob es sich bei den verschiedenen Disziplinen um Varianten einer Textsorte oder um verschiedene Textsorten oder Texttypen handelt.

An dieser Stelle sei kurz auf die Problematik der unterschiedlichen Verwendung der Begriffe Textsorte, Texttyp, Genre oder Konzept im deutschsprachigen Forschungsdiskurs verwiesen, ohne im Detail auf eine Diskussion der unterschiedlichen Begriffsverwendung eingehen zu können. Im vorliegenden Beitrag wird der Begriff Textsorte für den wissenschaftlichen Artikel verwendet. Gruber (2011) zählt einige wesentliche Faktoren auf, die für die Konstituierung einer Textsorte ausschlaggebend sind. Diese Faktoren stützen sich innerhalb der linguistischen Forschung auf einen breiten definitorischen Konsens: Textsorten haben eine spezifische Struktur, die den kommunikativen Zwecken einer bestimmten sozialen Gruppe dient. Sie präsentieren ihre Inhalte in einem für sie charakteristischen Stil und werden in bestimmten sozialen Situationen verwendet.

Für den deutschsprachigen wissenschaftlichen Artikel aus der Disziplin germanistische Linguistik lässt sich aus den erwähnten Untersuchungen ableiten, dass er zwar grundsätzlich der Textsorte wissenschaftlicher Artikel zugeordnet werden kann, dass er sich jedoch im Detail betrachtet von wissenschaftlichen Artikeln aus anderen Disziplinen unterscheidet. Worin genau diese Unterschiede bestehen und wie sie sich linguistisch festmachen lassen, wird bislang allerdings nur unzureichend beantwortet. Kaum Untersuchungen gibt es zudem zu Unterschieden innerhalb einer Disziplin, da die grundsätzliche Annahme besteht, dass sich wissenschaftliche Artikel innerhalb einer Disziplin kaum voneinander unterscheiden.

ISSN 1615-3014 
als Modell für den Unterricht wissenschaftlichen Schreibens

Diese Annahme wurde hier in einer ersten Pilotuntersuchung anhand der Einleitungssequenzen wissenschaftlicher Artikel verschiedener Subdisziplinen der germanistischen Linguistik überprüft. Dabei wurden 13 Texte nach dem Zufallsprinzip aus der Zeitschriftenschau der Zeitschrift für germanistische Linguistik (ZGL 2014) entnommen, die eine Sammlung wichtiger Aufsätze, die im Vorjahr erschienen sind, nennt. In einer Detailanalyse wurde die Makrostruktur dieser Einleitungssequenzen analysiert. Deren Gemeinsamkeiten und Unterschiede werden in diesem Artikel exemplarisch anhand von vier Beispieltexten diskutiert. Zusätzlich wurde zum Vergleich ein Text aus der Romanistik analysiert, der in eine der untersuchten Subkategorien Geschichte der Linguistik fällt.

Die Analyse folgt der unter Punkt 2 näher erläuterten Makrostruktur für deutschsprachige wissenschaftliche Artikel, die in Forschungsprojekten zum Thema wissenschaftliches Schreiben an der Universität Wien entwickelt wurde (vgl. Gruber et al. 2006; Gruber/Huemer/Rheindorf 2009; Huemer/Rheindorf/Gruber 2012) und die auf den für englischsprachige wissenschaftliche Artikel entwickelten Makrostrukturen von Swales und Feak (2009), Lewin, Fine und Young (2001) sowie auf systemisch funktionalen Textsortenanalysen (vgl. Halliday 2014; Eggins/Martin 1997) basiert.

Die Analyse der Einleitungssequenzen verschiedener Artikel aus Subdisziplinen der germanistischen Linguistik zeigt, dass sich die Strukturen der Einleitungssequenzen im Großen und Ganzen stark ähneln. Nur in der Subdisziplin Geschichte der Linguistik konnte in der Einleitungssequenz eine wesentlich andere Makrostruktur festgestellt werden. Dieser Unterschied und kleinere Abweichungen von der Makrostruktur werden im Folgenden detailliert beschrieben und es wird die Frage diskutiert, durch welche Faktoren sich eine andere Makrostruktur oder eine Abweichung ergeben.

\section{Theoretische Grundlagen und Analyseraster}

Für die Entwicklung der Kategorien zur Beschreibung der Makrostruktur deutschsprachiger wissenschaftlicher Artikel dienten als Vorlagen das sogenannte CARS Modell (Creating a Research Space) und das IMRD-Schema (Introduction - Methode - Results - Discussion) von Swales und Feak (2009) sowie eine Analyse der staging structure englischsprachiger Social Science Research Texts von Lewin, Fine und Young (2001). Swales und Feak haben für ihr mittlerweile sehr weit verbreitetes Modell (siehe Tabelle 1), das vor allem für den Unterricht studentischen Schreibens auf Englisch eingesetzt wird, die Einleitungssequenzen von 48 Artikeln aus den Fachbereichen Natur und Technik untersucht. Dabei diente Ihnen der professionelle Forschungsartikel (engl. 'research paper') als Vorlage für den Unterricht studentischen Schreibens (vgl. Swales/Feak 2009 und 2012). Die staging structure englischsprachiger Social Science Research Texts von Lewin, Fine und Young (siehe Tabelle 2) entstammt, wie der Name bereits sagt, der Analyse sozialwissenschaftlicher Artikel. Ihre qualitative Textanalyse orientiert sich an den Methoden der systemisch funktionalen Linguistik und ist insofern relevant, als sie sich ähnlicher Analysekategorien bedient, wie die in der Einleitung erwähnten Forschungsprojekte von Gruber et al.

Die Problematik der hier erwähnten anglo-amerikanischen Modelle liegt in ihrer mitunter wenig reflektierten Anwendung auf deutschsprachige wissenschaftliche Texte aller Disziplinen. Aufgrund sprachlicher und kulturspezifischer Unterschiede zwischen dem 
englischsprachigen und dem deutschsprachigen Wissenschaftsdiskurs sowie unterschiedlicher institutioneller Rahmenbedingungen an Universitäten verschiedener Kulturräume, können die Ergebnisse der anglo-amerikanischen Forschung nicht eins zu eins auf die deutschsprachige wissenschaftliche Textproduktion übertragen werden. Zudem kann man auch die deutschsprachige Wissenschaftslandschaft nicht als homogenes Gebilde betrachten, da auch Unterschiede zwischen deutschen, österreichischen und schweizerischen Universitäten bestehen. Es ist daher zumindest die Überprüfung anglo-amerikanischer Konzepte und deren Anpassung an den sprachlichen, situativen und kulturellen Kontext Voraussetzung für eine sinnvolle Analyse deutschsprachiger wissenschaftlicher Texte und deren Anwendung für den Unterricht studentischen Schreibens.

Im Rahmen von Forschungsprojekten zum Thema wissenschaftliches Schreiben an der Universität Wien wurden auf Basis der erwähnten anglo-amerikanischen Modelle deutschsprachige Kategorien für die Makrostruktur wissenschaftlicher Artikel und studentischer Arbeiten entwickelt (siehe Tabelle 3). Dabei wurden nicht nur Abstracts und Einleitungen analysiert, sondern auch die Makrostruktur von theoretischen Kapiteln, Methodenkapiteln, sowie Schlussteilen wissenschaftlicher Arbeiten. Die für die Analyse verwendeten Textsorten waren wissenschaftliche Artikel, Forschungsanträge, Forschungsexposés, studentische Haus- bzw. Seminararbeiten, Bachelorarbeiten, Masterarbeiten (und Diplomarbeiten) sowie Dissertationen vorwiegend geistes- und sozialwissenschaftlicher Fachbereiche (vgl. Gruber et al. 2006; Gruber/Huemer/Rheindorf 2009; Huemer/Rheindorf/Gruber 2012; Gruber/Huemer in Druck).

Für die Analyse der Makrostruktur der Texte, ihrer funktionalen Abschnitte und deren sprachliche Realisierungsvarianten wurden Kategorien der systemisch funktionalen Linguistik und Genreanalyse verwendet (vgl. Halliday 2014; Eggins/Martin 1997). Dafür wurden die Funktionen von Aussagen (engl. 'clauses') und deren Zugehörigkeit zu größeren funktionalen Abschnitten (engl. 'clause complexes') bestimmt. Durch Abstand oder Zeileneinzug visuell markierte Absätze im Text waren zudem ein Indikator für einen potentiellen neuen funktionalen Abschnitt, der jedoch anhand der Funktion sprachlicher Realisierungen einzelner Aussagen überprüft wurde.

Im Folgenden werden nun die beiden anglo-amerikanischen Modelle, die als Basis für die Analyse der deutschsprachigen Makrostruktur wissenschaftlicher Texte dienten, vorgestellt und verglichen. Tabelle (1) zeigt das CARS Modell von Swales und Feak (2009).

\begin{tabular}{|l|l|}
\hline Move/funktionaler Abschnitt & Sprachliche Realisierungsmöglichkeiten oder Handlungen \\
\hline Establishing a research territory. & $\begin{array}{l}\text { claiming centrality, and/or } \\
\text { placing your research within the field, and/or } \\
\text { reviewing items of previous research. }\end{array}$ \\
\hline Establishing a niche. & $\begin{array}{l}\text { counter-claiming, or } \\
\text { indicating a gap in current research, or } \\
\text { question raising, or } \\
\text { continuing a tradition. }\end{array}$ \\
\hline Occupying the niche. & $\begin{array}{l}\text { outlining purposes, or } \\
\text { announcing present research. } \\
\text { announcing principle findings. }\end{array}$ \\
\hline
\end{tabular}


indicating research article structure.

Tabelle 1: Das CARS Modell von Swales und Feak (2009)

Tabelle (2) zeigt die Struktur von social sciences research texts von Lewin, Fine und Young (2001).

\begin{tabular}{|l|l|l|}
\hline $\begin{array}{l}\text { Move/funktionaler } \\
\text { Abschnitt }\end{array}$ & Obligatorische Handlungen & Fakultative Handlungen \\
\hline Claiming relevance of field. & $\begin{array}{l}\text { Asserting relevance of field } \\
\text { of which research is part } \\
\text { of. } \\
\text { Reporting what is known } \\
\text { about phenomena under } \\
\text { study. }\end{array}$ & $\begin{array}{l}\text { Making assertions about the } \\
\text { research process of others. } \\
\text { Reporting terminology } \\
\text { conventions. } \\
\text { Reporting conclusions drawn by } \\
\text { previous authors. } \\
\text { Drawing [own] conclusions } \\
\text { about the research of others. } \\
\text { Metacomments. } \\
\text { Narrowing parameters of field. }\end{array}$ \\
\hline $\begin{array}{l}\text { Establishing the gap present } \\
\text { research is meant to fill. }\end{array}$ & $\begin{array}{l}\text { Pointing out deficiencies in } \\
\text { the present state of } \\
\text { knowledge. }\end{array}$ & $\begin{array}{l}\text { Positing an ideal way to fill the } \\
\text { gap that has just been created. } \\
\text { Mitigating - Pointing out the } \\
\text { positive contribution of the } \\
\text { previous research. }\end{array}$ \\
\hline $\begin{array}{l}\text { Previewing authors' new } \\
\text { accomplishments. }\end{array}$ & $\begin{array}{l}\text { Stating purpose of present } \\
\text { study or contents of article. }\end{array}$ & $\begin{array}{l}\text { Positing an ideal way to fill the } \\
\text { gap that has just been created. } \\
\text { Reporting what is known about } \\
\text { phenomena under study. } \\
\text { Justifying hypothesis. } \\
\text { Disclosing whether hypothesis } \\
\text { has been confirmed or not. } \\
\text { Summarizing methods. } \\
\text { Presenting hypothesis or } \\
\text { research questions. }\end{array}$ \\
\hline
\end{tabular}

Tabelle 2: Struktur von social sciences research texts von Lewin, Fine und Young (2001)

Vergleicht man die beiden anglo-amerikanischen Modelle, so zeigt sich, dass sie sich nur in wenigen Punkten voneinander unterscheiden. Sowohl für Swales und Feak als auch für Lewin, Fine und Young besteht die Makrostruktur einer Einleitung englischsprachiger wissenschaftlicher Artikel aus den genannten Disziplinen aus drei moves oder Abschnitten. Auch wenn im CARS Modell andere Bezeichnungen für diese drei Abschnitte gewählt wurden als im Modell für social science research texts, so benennen sie doch Ähnliches, wenn nicht sogar in manchen Fällen Gleiches. Während Swales und Feak als ersten funktionalen Abschnitt establishing a research territory nennen, bezeichnen Lewin, Fine und Young diesen Abschnitt als claiming relevance of field. In beiden Fällen steht das Forschungsfeld oder Territorium im Zentrum. Im ersten Fall wird es etabliert, im zweiten Fall seine Relevanz behauptet. Das Etablieren ist eine neutralere Handlung als das Behaupten von 
Relevanz, allerdings führen Swales und Feak bei den möglichen Realisierungsvarianten dieses Abschnitts ebenfalls das „Behaupten von Zentralität“ an. Damit wird also deutlich, dass sich diese beiden Funktionen, auch wenn sie von den unterschiedlichen AutorInnengruppen etwas anders benannt wurden, kaum voneinander unterscheiden. Ähnlich verhält es sich mit den jeweils zweiten und dritten Abschnitten der beiden Modelle: establishing a niche und occupying the niche bei Swales und Feak oder establishing the gap present research is meant to fill und previewing authors' new accomplishments bei Lewin, Fine und Young. Auch hier unterscheiden sich die von den AutorInnen gewählten Bezeichnungen der Abschnitte in ihrer Bedeutung nur gering voneinander. Während bei Swales und Feak eine Nische auch relativ neutral hergestellt werden kann, indem an eine Forschungstradition angeknüpft wird, wird bei Lewin, Fine und Young der Mangel oder die Forschungslücke betont, der beseitigt oder die gefüllt werden soll. Die Nische wird bei Swales und Feak dann schlussendlich mit ähnlichen Handlungen besetzt wie die etwas neutraler formulierte Vorschau auf die Leistungen der Autorin/des Autors bei Lewin, Fine und Young. In beiden Fällen geben die AutorInnen an, dass in den Texten der Zweck der Studie genannt wird bzw. genannt werden sollte, wobei Lewin, Fine und Young hier etwas mehr ins Detail gehen und von einer Vorschau auf die Studie mit konkreter Nennung von Hypothesen, Forschungsfragen und Methoden sprechen. Swales und Feak halten es zudem noch für relevant zu erwähnen, dass in den Texten die wichtigsten Ergebnisse genannt werden (sollen) und die Artikelstruktur angekündigt wird.

Der wesentlichste Unterschied zwischen diesen beiden Modellen besteht darin, dass Lewin, Fine und Young zwischen obligatorischen und fakultativen Realisierungsmöglichkeiten innerhalb eines Abschnitts unterscheiden und dass sie eine größere Anzahl von Realisierungsmöglichkeiten sowie eine teilweise detailliertere Beschreibung von Varianten anbieten.

Tabelle (3) zeigt nun die für deutschsprachige wissenschaftliche Artikel angepasste Makrostruktur von Einleitungen. Die kursiv markierten sprachlichen Realisierungsmöglichkeiten sind im Falle des Vorkommens dieses Abschnittes obligatorisch. Die anderen Realisierungsmöglichkeiten können als fakultativ betrachtet werden.

\begin{tabular}{|l|l|}
\hline Move/funktionaler Abschnitt & Sprachliche Realisierungsmöglichkeiten \\
\hline $\begin{array}{l}\text { An bestehende Forschung } \\
\text { anknüpfen. }\end{array}$ & $\begin{array}{l}\text { Das zu untersuchende Phänomen beschreiben. } \\
\text { Hintergründe darstellen. } \\
\text { Stand der Wissenschaft skizzieren. }\end{array}$ \\
\hline $\begin{array}{l}\text { Einen Mangel ankündigen, den die } \\
\text { Arbeit beseitigen soll. }\end{array}$ & $\begin{array}{l}\text { Einen Mangel im derzeitigen Stand des Wissens } \\
\text { aufzeigen. } \\
\text { Den Beitrag früherer Studien erwähnen. }\end{array}$ \\
\hline Die Relevanz der Arbeit behaupten. & $\begin{array}{l}\text { Begründen warum die Arbeit/der eigene Beitrag von } \\
\text { Bedeutung ist oder } \\
\text { allgemein die Relevanz des Untersuchungsfeldes } \\
\text { aufzeigen. }\end{array}$ \\
\hline $\begin{array}{l}\text { Die Leistungen der AutorIn } \\
\text { ankündigen. }\end{array}$ & $\begin{array}{l}\text { Den Zweck oder die Ziele der Arbeit anführen } \\
\text { Das Untersuchungsfeld einschränken. } \\
\text { Die Forschungsfragen/Hypothesen präsentieren. }\end{array}$ \\
\hline
\end{tabular}




\begin{tabular}{|l|l|}
\hline & $\begin{array}{l}\text { Auf Theorien/Methoden/Daten/Vorgehensweise } \\
\text { verweisen. } \\
\text { Die wichtigsten Ergebnisse vorwegnehmen. }\end{array}$ \\
\hline Die Vorgehensweise skizzieren. & Den Aufbau des Textes ankündigen. \\
\hline
\end{tabular}

Tabelle 3: Die Makrostruktur deutschsprachiger Artikel

Die Analyse der deutschsprachigen Texte ergab (vgl. Gruber et al. 2006; Gruber/Huemer/Rheindorf 2009; Huemer/Rheindorf/Gruber 2012; Gruber/Huemer in Druck), dass sich die Einleitung wissenschaftlicher Arbeiten allgemein in fünf verschiedene funktionale Abschnitte unterscheiden lässt, wobei in professionellen wissenschaftlichen Artikeln der letzte funktionale Abschnitt „die Vorgehensweise skizzieren“ häufig entfällt. Dies kann mit der Kürze wissenschaftlicher Artikel begründet werden, die entgegen umfangreicherer Monografien oder studentischer Abschlussarbeiten, nicht unbedingt eine Vorausschau auf die Kapitelstruktur zum Zweck der LeserInnenorientierung verlangen.

Der erste funktionale Abschnitt ,an bestehende Forschung anknüpfen“ ist dem ersten Abschnitt in den beiden englischsprachigen Modellen sehr ähnlich, obwohl er in den bereits erwähnten Untersuchungen deutschsprachiger wissenschaftlicher Arbeiten eher der von Lewin, Fine und Young bezeichneten obligatorischen Handlung reporting what is known about phenomena under study entspricht. Dies könnte daran liegen, dass es sich bei den untersuchten deutschsprachigen Texten ebenso wie bei Lewin, Fine und Young hauptsächlich um Arbeiten aus dem Bereich Sozial- und Geisteswissenschaften handelte.

Der von Swales und Feak benannte zweite Abschnitt establishing a niche und von Lewin, Fine und Young bezeichnete Abschnitt establishing the gap present research is meant to fill wird in deutschsprachigen wissenschaftlichen Artikeln häufig in die zwei verschiedenen funktionalen Abschnitte einen Mangel ankündigen, den die Arbeit beseitigen soll und die Relevanz der Arbeit behaupten getrennt. Diese Trennung in zwei Abschnitte wird in deutschsprachigen Texten bisweilen durch Absätze markiert. Ein Mangel wird angekündigt, indem ein Mangel im derzeitigen Stand des Wissens aufgezeigt wird, wobei es nützlich sein, kann die Beiträge früherer Studien zu erwähnen, die zur Beseitigung dieses Mangels bereits beigetragen haben. Die Relevanz der Arbeit wird behauptet, indem begründet wird, warum die Arbeit von Bedeutung ist. Eventuell wird zusätzlich die generelle Relevanz des Untersuchungsfeldes aufgezeigt. Nicht immer werden beide Abschnitte realisiert, um der Arbeit Bedeutung zu verleihen.

Relativ ähnlich zum dritten funktionalen Abschnitt des CARS Modells und der Struktur von Social Science Research Texts ist wiederum der vierte Abschnitt dieses Modelles die eigenen Leistungen ankündigen. In diesem Abschnitt wird der Zweck oder werden die Ziele der Arbeit angeführt. Eventuell schränkt die Autorin/der Autor zusätzlich das Untersuchungsfeld ein, präsentiert Forschungsfragen und Hypothesen, verweist auf Theorien, Methoden, Daten und Vorgehensweise und nimmt eventuell die wichtigsten Ergebnisse vorweg.

Insgesamt betrachtet unterscheidet sich die Makrostruktur der Einleitung der in den erwähnten Forschungsprojekten untersuchten deutschsprachigen wissenschaftlichen Texte nur gering von der Makrostruktur der anglo-amerikanischen Modelle. Abgesehen von der 
Formulierung sinnvoller deutschsprachiger Kategorien für die jeweiligen funktionalen Abschnitte waren nur geringe Anpassungen notwendig.

\section{Qualitative Analyse der Einleitungssequenzen: Eine Pilotstudie}

In der hier dargestellten Pilotstudie wurde die in Tabelle (3) beschriebene Makrostruktur nun anhand deutschsprachiger wissenschaftlicher Artikel aus der Disziplin germanistische Linguistik überprüft und es wurde untersucht, ob die Makrostruktur mancher Subdisziplinen der germanistischen Linguistik, wie z. B. Artikel mit stärker literaturwissenschaftlich oder geschichtswissenschaftlich orientierten Fragestellungen, von dieser Struktur abweicht. Die Disziplin germanistische Linguistik ist insbesondere deshalb interessant, da sie ein breites Spektrum an Subdisziplinen, Fragestellungen und methodischen Zugängen zur Beantwortung von Forschungsfragen aufweist. Sie eignet sich daher besonders gut für eine Untersuchung von Varianten innerhalb einer Disziplin.

Für die qualitative Analyse der Einleitungssequenzen wissenschaftlicher Artikel wurden 13 Artikel aus verschiedenen Subdisziplinen der germanistischen Linguistik ausgewählt und analysiert $^{1}$. Die Texte wurden aus der Zeitschriftenschau der Zeitschrift für germanistische Linguistik entnommen, die eine Sammlung wichtiger Aufsätze, die im Vorjahr erschienen sind, nennt (ZGL 2014). Diese Übersicht enthält 51 verschiedene Kategorien, nach denen Artikel im Fach germanistische Linguistik gruppiert werden und die hier als Subdisziplinen bezeichnet werden können. Darunter werden u. a. Computerlinguistik, Deutsch als Fremdoder Zweitsprache, Geschichte der Linguistik, Morphologie, Sprachgeschichte, Syntax, Wissenschaftstheorie und Wortbildung genannt.

In diesem Artikel wird je eine Einleitungssequenz aus der Kategorie oder Subdisziplin Sprachgeschichte, Wortbildung, Deutsch als Fremd- oder Zweitsprache und Geschichte der Linguistik (in dieser Reihenfolge) exemplarisch im Detail besprochen, um die Ähnlichkeiten $\mathrm{zu}$ und die Unterschiede von der in diesem Artikel vorgestellten Makrostruktur zu verdeutlichen. Zusätzlich wird zum Vergleich ein weiterer Artikel aus der Kategorie Geschichte der Linguistik aus der Romanistik analysiert und besprochen.

In den folgenden Abschriften der analysierten Einleitungssequenzen werden von der Autorin eingefügte Textkommentare und Analysen kursiv und in eckigen Klammern dargestellt.

Der erste Artikel stammt aus der Subdisziplin Sprachgeschichte und trägt den Titel „Zwischen Transparenz und Lexikalisierung: Das Wortbildungsmuster X-ung(e) im Mittelhochdeutschen“. Er wurde von Stefan Hartmann verfasst und 2013 in der Zeitschrift Beiträge zur Geschichte der deutschen Sprache und Literatur veröffentlicht. Diese Einleitung folgt dem klassischen Muster deutschsprachiger wissenschaftlicher Artikel aus dem Bereich der Geistes- und Sozialwissenschaften und ist ein prototypisches Beispiel für die Einleitungssequenzen der meisten anderen untersuchten Artikel.

Die Einleitung beginnt mit einer Beschreibung des zu untersuchenden Phänomens und einer darauf folgenden Diskussion des aktuellen Stands der Wissenschaft zu diesem Phänomen.

\footnotetext{
1 Alle analysierten Artikel werden am Ende dieses Artikels unter der Überschrift Korpus alphabetisch aufgelistet. Zusätzlich wird bei jedem Artikel angegeben, in welcher Kategorie der Zeitschriftenvorschau der Zeitschrift für germanistische Linguistik, bzw. in welcher Subdisziplin er erschienen ist.
} 
Anschließend wird die These präsentiert, die Untersuchungsmethode kurz erwähnt und, ähnlich wie in einem Abstract, werden die wichtigsten Ergebnisse angeführt. Es folgt eine Begründung der Relevanz der Arbeit und eine noch etwas genauere Beschreibung der methodischen Vorgehensweise. Abgeschlossen wird die Einleitung mit einem weiteren vorweggenommenen wichtigen Ergebnis der Untersuchung.

\section{Beispiel (1)}

$1 \quad$ Einleitung

[Das zu untersuchende Phänomen beschreiben] Nomina auf -ung begegnen uns im Neuhochdeutschen quasi auf Schritt und Tritt. Okkasionelle Bildungen wie HandySprachsteuerung, Klarifizierung, Kronenvergreisung, Kurzdiffamierung oder Produktbejahung ${ }^{1}$ zeigen, dass das Suffix - ung aus der Alltags- wie aus den Fachsprachen der Gegenwart kaum wegzudenken ist. Exemplarisch seien nur die Finanz- und Wirtschaftssprache (Eurorettung, Wachstumsbeschleunigung), die Sprache der Politik (Übergangsregierung) und die Techniksprache (Fernsteuerung) genannt. Bei diesen vergleichsweise jungen Bildungen handelt es sich aber - mit Ausnahme des wohl als Lehnformung zu engl. clarification gebildeten Begriffs Klarifizierung - durchweg um Komposita mit längst bestehenden und sehr geläufigen, mithin tokenfrequenten -ungBildungen als Zweitglied. Indes ist die morphologische Produktivität des Wortbildungsmusters X-ung, verstanden als Größe der Anwendungsdomäne eines Wortbildungsmusters, deutlich zurückgegangen (vgl. Demske 2000; zu unterschiedlichen Produktivitätsbegriffen vgl. Rainer 1987).

[Stand der Wissenschaft skizzieren] Definiert man mit Scherer (2006) Wortbildungswandel als Wandel von Wortbildungsbeschränkungen, kann der Produktivitätswandel der -ungNominalisierung als Paradebeispiel für genuinen Wortbildungswandel - in Abgrenzung von mancherlei Schnittstellenphänomenen - gelten. Folgerichtig sind es die Restriktionen für Neubildungen auf -ung, die in der jüngeren Forschungsliteratur sowohl in synchroner wie auch in diachroner Perspektive auf besonderes Interesse stießen (diachron Demske 1999, 2000, 2002; synchron mit einigen diachronen Exkursen Ehrich/Rapp 2000; Ehrich 2002; rein synchron Roßdeutscher 2010; Roßdeutscher/Kamp 2010). Die höchst unterschiedlichen Herangehensweisen dieser Arbeiten - man vergleiche etwa den sortensemantischdekompositionalen Ansatz Ehrich/Rapps mit Roßdeutschers Analyse auf Grundlage der Diskursrepräsentationstheorie - mögen den Eindruck einer »unbefriedigenden und widersprüchlichen Beschreibung der -ung-Nomina in der Literatur« erwecken, wie ihn Knobloch (2002: 333) beklagt. Diese etwas plakative Behauptung muss freilich in zweifacher Hinsicht relativiert werden. Zum einen kann die Heterogenität der Beschreibungs- und Erklärungsansätze durchaus auch als Gewinn aufgefasst werden, insofern sich die unterschiedlichen Positionen gegenseitig hinterfragen und somit daran erinnern, dass gerade im Bereich der Semantik absolute Wahrheiten kaum formuliert werden können. Zum anderen ist diese Widersprüchlichkeit auch deshalb höchst instruktiv, weil sie ja aus der von Knobloch (2002) ebenfalls erkannten Uneinheitlichkeit der Wortbildungsprodukte selbst resultiert.

[(Hypo)these präsentieren, Methode anführen und die wichtigsten Ergebnisse vorwegnehmen] Die vorliegende Untersuchung vertritt die These, dass eben diese Diversifikation des Outputs bereits im Mhd. ihren Anfang nahm und wesentlich für die Verschärfung der Inputrestriktionen mitverantwortlich ist. Anhand einer Korpusanalyse wird gezeigt, dass die ung-Bildungen des Mhd. erwartungsgemä $\beta$ deutlich verbnäher sind als die tokenfrequentesten -ung-Derivate des Gegenwartsdeutschen, dass aber gerade im Bereich der häufig verwendeten 
theologischen und juristischen Fachbegriffe erste Lexikalisierungstendenzen und damit einhergehend ein Rückgang der semantischen Transparenz unübersehbar sind.

[Begründen warum die Arbeit von Bedeutung ist] Während bisherige diachrone Untersuchungen zum -ung-Suffix beim Frühnhd. ansetzen, nimmt diese Arbeit m.W. erstmalig die Situation im Mhd. ausführlich in den Blick. [Auf Methoden/Daten verweisen] Zu diesem Zweck wurde ein Korpus erstellt, das nach einem kurzen Überblick zur Etymologie und Entwicklung des Wortbildungsmusters X-ung (2) zunächst vorgestellt und mit Blick auf Umfang und Repräsentativität kritisch gewürdigt wird (3). Es folgen eine semantische (4) und eine syntaktische (5) Analyse der Korpusdaten, wobei im Rahmen der semantischen Analyse das hier vertretene Konzept der Verbnähe bzw. -ferne vorgestellt wird [Die wichtigsten Ergebnisse vorwegnehmen] und die Lexikalisierungstendenzen der Wortbildungsprodukte auf -ung als Wandel zu mehr $>$ Nominalität $<$ gedeutet werden.

(Hartmann 2013: 159-161)

Der zweite Artikel wurde von Petra Szatmári (2011) in der Subdisziplin Wortbildung verfasst, trägt den Titel „Affixoide - Pro und Kontra“" und stammt aus dem Jahrbuch der ungarischen Germanistik. Dieses Beispiel wurde ausgewählt, da es prototypisch für Artikel ist, die ein Thema theoretisch und nicht empirisch aufarbeiten. Auch hier werden alle obligatorischen Abschnitte der dargestellten Makrostruktur realisiert, auch wenn in diesem Fall im Abschnitt die Leistungen der Autorin ankündigen naturgemäß kein Verweis auf die verwendeten Methoden stattfinden oder Hypothesen formuliert werden können (s. dazu auch Doleschal/Scherling in diesem Heft).

Mit nur einem einzigen Satz führt die Autorin in das Thema ein, indem sie das zu untersuchende Phänomen beschreibt. Darauf folgt ein stark komprimierter Abriss des aktuellen Stands der Wissenschaft, dessen ausführliche Diskussion das eigentliche Thema des Beitrags stellt. Wiederum mit nur einem einzigen Satz begründet die Autorin die Relevanz ihres Beitrags bevor sie ihre Einleitung mit der Beschreibung des Ziels der Arbeit und einem Verweis auf die Vorgehensweise schließt.

Beispiel (2)

1 Einführendes

[Das zu untersuchende Phänomen beschreiben] Als besonders produktive Wortbildungstypen erweisen sich im Deutschen die Komposition, die explizite Derivation ${ }^{1}$ und die Konversion (vgl. Donalies 2007b: 6). [Stand der Wissenschaft skizzieren] Die intensivere Beschäftigung mit Wortbildungsprozessen in den 1960er/1970er Jahren rückte ein Phänomen, das sich zwischen den beiden wichtigsten Wortbildungsarten im Deutschen, der Komposition und der Derivation, bewegt, immer mehr ins Blickfeld und veranlasste Fleischer ( ${ }^{3} 1974$ : 70$)$ von Affixoiden (Präfixoiden, Suffixoiden) zu sprechen. $^{2}$ Mit ihrer „Ausgliederung“ als Übergangsphänomen setzte zugleich die wissenschaftliche Diskussion dieses Begriffs ein und führte in der Folge dazu, dass Fleischer selbst den Begriff verwarf (vgl. Fleischer; Barz ${ }^{2}$ 1995). [Begründen warum die Arbeit von Bedeutung ist] Dennoch, auch wenn einige Autoren suggerieren wollen, dass der Begriff von der Fachwelt zur Gänze verworfen worden ist, ${ }^{3}$ verblieben genügend Befürworter, so dass die Auseinandersetzung in keinster Weise als abgeschlossen angesehen werden kann.

[Ziel der Arbeit anführen und auf Vorgehensweise verweisen] Im Folgenden soll der neuere Stand der Diskussion umrissen werden, wobei gelegentlich, auch wenn es hier in erster Linie gemäß der Ausschreibung um Reflexionen auf die Fachliteratur der letzten Dekade geht, auf 
als Modell für den Unterricht wissenschaftlichen Schreibens

länger zurückliegende theoretische und empirische Forschungsergebnisse zurückgegriffen wird, um den Hintergrund für aktuellere Untersuchungen einleuchtender zu erfassen.

(Szatmári 2011: 156-157)

Der dritte Artikel aus der Subdisziplin Deutsch als Fremd- oder Zweitsprache wurde von Andrea Bicsár verfasst und trägt den Titel „Kribbeln im Bauch: Emotionen im Deutschen als Fremdsprache. Eine empirische Studie“. Der Artikel ist 2012 ebenfalls in der Zeitschrift Jahrbuch der ungarischen Germanistik erschienen. Das Beispiel wurde hier zur Veranschaulichung ausgewählt, da es wie manche andere der untersuchten Artikel Abweichungen von der Makrostruktur aufweist. D. h. es werden entweder nicht alle relevanten obligatorischen Abschnitte der Makrostruktur realisiert und/oder die funktionalen Abschnitte werden nicht in der üblichen Reihenfolge präsentiert.

Eine erste Präsentation von Forschungsfragen, die eher allgemein gehalten sind, wird in die Beschreibung des zu untersuchenden Phänomens eingebettet, bzw. dazu verwendet, diese zu illustrieren. Anschließend wird das Ziel der Arbeit kurz präsentiert und es wird auf die verwendete Methode verwiesen. Relativ ausführlich werden in der Folge die konkreten Forschungsfragen und die Hypothese präsentiert sowie auf Theorien verwiesen, die für die Hypothesenbildung ausschlaggebend waren. Dafür wird jedoch darauf verzichtet, den aktuellen Stand der Wissenschaft zu skizzieren und zu begründen warum die Arbeit von Bedeutung ist. Die beiden zuletzt genannten funktionalen Abschnitte sind zentrale Elemente einer Einleitung und verschaffen einem wissenschaftlichen Artikel stärkeres argumentatives Gewicht. Offensichtlich war hier jedoch das Fehlen dieser Abschnitte kein Hinderungsgrund für die erfolgreiche Publikation des Artikels.

Beispiel (3)

[Das zu untersuchende Phänomen beschreiben] „Die Sprache ist die Kleidung der Gedanken“, fasst Samuel Johnson, der englische Lexikograf, Schriftsteller, Dichter und Kritiker die Funktion von Sprache zusammen (1905: 181). Doch Sprache eignet sich nicht nur zum Ausdruck der Gedanken, sondern befähigt uns, unsere Emotionserfahrung verbal zu vermitteln. [Forschungsfragen präsentieren] Wie setzen wir Sprache ein, um über Gefühlserlebnisse zu berichten? Diese Frage ist noch spannender bei Sprechern, die ihre Freude, Überraschung oder Aufregung über ein Ereignis nicht in ihrer Muttersprache, sondern in einer Fremdsprache beschreiben möchten. Wie verbalisieren L2-Lerner Gefühlszustände in der Zielsprache?

[Ziel der Arbeit anführen und auf Methode verweisen] Der vorliegende Aufsatz setzt sich zum Ziel, den lernersprachlichen Gebrauch emotionsbezeichnender Sprachelemente, d. h. jenes Segments des Gefühlswortschatzes der Zielsprache im Rahmen einer empirischen Untersuchung $\mathrm{zu}$ ermitteln, das Lexeme umfasst, die explizit auf bestimmte Emotionen referieren (vgl. z. B. Schwarz-Friesel 2007: 144; Konstantinidou 1997: 84; Kövecses 2000: 2). Dabei werden Tendenzen der Emotionsbeschreibung im sprachlichen Output ungarischer Deutsch-als-Fremdsprache-Sprecher (DaF-Sprecher) im Vergleich zu Realisationsmustern deutscher Muttersprachler bewertet. [Forschungsfragen präsentieren] Die Arbeit geht zwei primären Leitfragen nach: 1) Welche grundlegenden Unterschiede sind auf der Ebene der Lexik in der lernersprachlichen bzw. muttersprachlichen Kommunikation über Gefühle im Deutschen zu erkennen? und 2) Inwiefern trägt der Aufenthalt im Zielland zur normgerechten Emotionsrealisation in der Zielsprache bei? [Hypothese präsentieren] Die Studie postuliert, dass DaF-Lerner, die die Möglichkeit haben, ihre L2 in natürlichen Interaktionen im Zielland 
bzw. mit Muttersprachlern zu verwenden, Emotionen kompetenter versprachlichen als Lerner, die ihre Fremdsprache primär im gesteuerten Unterricht gebrauchen. [Auf Theorien verweisen] Welche Erkenntnisse dienen als Grundlage für die Annahme, dass Fremdsprachensprecher Emotionen in ihrer Zielsprache ggf. anders verbalisieren als Muttersprachler der L2 und der natürliche Kontakt zur L2 die „emotionale Kompetenz“ in der Fremdsprache fördert? Den Ausgangspunkt der Hypothese bilden Folgerungen der kontrastiven Linguistik und der Psycholinguistik bzw. Überlegungen aus der Fremdsprachenerwerbsforschung, die darauf hindeuten, dass der verbale Emotionsausdruck bedeutende interlinguale und interkulturelle Unterschiede aufweist und damit eine aufschlussreiche Forschungsperspektive aus der Sicht der Lernersprache bildet. Bevor diese jedoch eingehend dargestellt werden können, verlangt die Frage Aufmerksamkeit, was wir unter emotionsbezeichnenden Sprachelementen verstehen.

(Bicsár 2012: 179-180)

Der vierte Artikel aus der Subdisziplin Geschichte der Linguistik stammt aus einem Beitrag von Barbara Kaltz (2013), trägt den Titel „Zwischen den Stühlen. Paul Lévy und die französische Germanistik in der ersten Hälfte des 20. Jahrhunderts." und wurde in der Zeitschrift Historiographica linguistica veröffentlicht. Die hier analysierte Einleitung weist große Abweichungen von der Makrostruktur der anderen Texte auf. Aus diesen Abweichungen kann geschlussfolgert werden, dass es sich hier um eine alternative Makrostruktur handelt, welche weder mit der von Swales und Feak, noch mit der von Levin, Fine und Young, noch mit der für deutschsprachige wissenschaftliche Artikel erstellten, übereinstimmt.

Wie auf den ersten Blick bereits zu erkennen ist, ist diese Einleitung um einiges kürzer als die der anderen Artikel. Die Analyse zeigt zudem, dass sie sich auch hinsichtlich der Realisierung relevanter funktionaler Abschnitte deutlich von den anderen unterscheidet. Es wird hier lediglich das zu untersuchende Phänomen kurz beschrieben, das in einen historischen Kontext eingebettet wird (In der ersten Hälfte des 20. Jahrhunderts). Nur implizit weist der Text auf einen Mangel in der bestehenden Forschung und die Leistungen der Autorin hin (Verfasst wurden sie von einem heute weitgehend vergessenen...). Der Mangel und die Leistungen der Autorin werden jedoch sprachlich nicht ausführlich dargestellt und als eigener funktionaler Abschnitt markiert, sowie es in den anderen Texten geschieht.

Beispiel (4)

\section{Einleitung}

[Beschreiben des zu untersuchenden Phänomens] In der ersten Hälfte des 20. Jahrhunderts erschienen zwei wichtige Werke zur Geschichte des Deutschen in Frankreich, die beide in methodischer Hinsicht Ferdinand Brunot (1860-1938) und seiner großen Historie de la langue française des origines à 1900 verpflichtet sind, eine umfassende Sprachgeschichte des Elsass und Lothringens bis 1918 und eine Untersuchung zur Einwirkung und Verbreitung des Deutschen in Frankreich von den Anfängen bis 1944. Verfasst wurden sie von einem heute weitgehend vergessenen französischen Germanisten namens Paul Lévy (Lévy 1929, 1950, 1952).

(Kaltz 2013: 439)

Zur Untermauerung der Annahme, dass es sich bei dieser Abweichung von der Makrostruktur in der Subkategorie Geschichte der Linguistik weder um eine Ausnahme noch um ein rein disziplinspezifisches Phänomen handelt, sei noch ein weiteres Beispiel (5) hier angeführt, das in dieselbe Kategorie fällt, jedoch im Bereich Romanistik angesiedelt ist. 
Die Einleitung des von Franz-Josef Klein verfassten Artikels mit dem Titel „Zur Darstellung logographischer und phonographischer Schriftsysteme bei José de Acosta und Gregorio Garcí“, der 2009 ebenfalls in der Zeitschrift Historiographia Linguistica erschienen ist, hat zwar eine übliche Länge und mehr als einen funktionalen Abschnitt, geht jedoch ähnlich vor wie das Beispiel (4): Der Fokus liegt auf der zeitlichen Markierung (Seit mehreren Jahrzehnten) und der Einbettung in einen historischen Kontext, auch wenn hier relevante funktionale Abschnitte geschickt mit dieser historischen Einbettung verknüpft wurden.

Beispiel (5)

1. Einleitung

[An bestehende Forschung anknüpfen] Seit mehreren Jahrzehnten gehört die Sprachbetrachtung im spanischen Siglo de Oro $\mathrm{zu}$ den beliebtesten Themen der Wissenschaftshistoriographie im Bereich der Romanistik. Besonderes Interesse haben hierbei immer wieder die zahlreichen Texte aus jener Zeit gefunden, die sich der Normierung der spanischen Orthographie widmen (Überblick u.a. bei Rosenblat 1951, Esteve Serrano 1977, Weißkopf 1994, Meisenburg 1996). [Einen Mangel ankündigen, den die Arbeit beseitigen soll] Demgegenüber ist bisher weitgehend unbeachtet geblieben, dass im Spanien des 16. und 17. Jahrhunderts auch bereits die Funktionsweise von Schrift im Allgemeinen sowie die unterschiedlichen Schriftsysteme (Logographie vs. Phonographie) diskutiert werden. Ebenso werden schon Überlegungen zur Problematik der Entstehung der Schrift angestellt. Die vielen, mittlerweile von der Forschung recht gut aufgearbeiteten spanischen Grammatiken des Siglo de Oro und die noch zahlreicheren Orthographietraktate sind allerdings nicht der Ort, an dem diese Diskussion stattfindet. Diese Werke verfolgen meist praktische, d. h. sprachnormative oder didaktische Ziele, für die die Reflexion grundlegender Fragen der oben beschriebenen Art nicht relevant ist. Hinzu kommt, dass die Autoren der spanischen Grammatiken in den meisten Fällen in der Tradition der europäischen Alphabetschriften befangen sind. Sie kennen die (zeitgenössischen oder vergangenen) Kulturen Asiens oder Amerikas im Allgemeinen nicht aus eigener Anschauung und kommen auch deshalb selten auf den Gedanken, über andere Schriftsysteme als das alphabetische nachzudenken.

[Die Leistungen der AutorIn ankündigen] Für die hier zu behandelnde Thematik relevant ist hingegen (wenn auch nur in Einzelfällen) eine andere Sorte von Texten. Es sind dies die zahlreichen Berichte über die Neue Welt, die im 16. und 17. Jahrhundert in großer Zahl (meist in Amerika selbst) verfasst und als Cartas de relación, Crónicas de Indias, Historias de Indias oder unter noch anderen Bezeichnungen publiziert werden (Überblick z. B. bei Mignolo 1982: 57-116). Hinzu kommen verschiedene Typen hierauf aufbauender Texte historischen oder philosophischen Charakters. Bisher wurden diese frühen "Kolonialchroniken" (Gumbrecht 1987: 227) von der Wissenschaft eher marginal zur Kenntnis genommen (vgl. Alvar [1992: 59]: "poco se han preocupado por estos temas los filólogos españoles").

(Klein 2009: 1-2)

\section{Diskussion der Ergebnisse}

Durch die vorliegende Pilotstudie konnte festgestellt werden, dass sich die Makrostruktur von Einleitungen im Fach germanistische Linguistik vorwiegend an dem klassischen Muster geistes- und sozialwissenschaftlicher Texte orientiert. Dies scheint sich daraus zu ergeben, dass in den meisten hier untersuchten Artikeln empirische Methoden zur Bearbeitung der Fragestellung verwendet wurden, in denen entweder 1) Daten erhoben und analysiert wurden, 
2) bereits erhobene Daten analysiert wurden oder 3) Theorien, die aus der Untersuchung von erhobenen und analysierten Daten entstanden sind, besprochen und diskutiert wurden. Größere Abweichungen von dieser Makrostruktur werden dann festgestellt, wenn sich der Artikel einer anderen Methode oder Vorgehensweise bedient, sowie in dem Beitrag von Barbara Kaltz aus der Subdisziplin Geschichte der Linguistik (Beispiel 4) oder dem Artikel von Franz-Josef Klein (Beispiel 5). Beide Artikel haben eine vorrangig historische Fragestellung, die nicht mit einer auf empirischen Daten basierenden Analyse bearbeitet werden kann, sondern für die eine historische Einbettung und Aufbereitung des Themas zentral ist. Diese Abweichungen von der klassischen Makrostruktur in der Subkategorie Geschichte der Linguistik können als alternatives Muster interpretiert werden und sollten nicht als Abweichung von einer Norm verstanden werden.

Abweichungen, wie sie in der Subdisziplin Wortbildung des von Petra Szatmári verfassten Artikels auftreten (Beispiel 2) werden damit begründet, dass in diesem Artikel eine Theorieoder Literaturbesprechung das zentrale Thema stellt. Das heißt ähnlich wie in den Beispielen aus der Subdisziplin Geschichte der Linguistik steht hier eine im historischen Kontext eingebettete theoretische Diskussion bestehender Forschung im Vordergrund. Ähnliche Abweichungen, die sich durch die Wahl des Themas begründen lassen und die somit auch auf die methodische Vorgehensweise Einfluss haben, finden sich auch noch in weiteren der 13 untersuchten Artikeln. So wird z. B. in dem Artikel von Beißwenger und Lemnitzer 2013, erschienen im Journal for Language Technology and Computational Linguistics, der aktuelle Stand der Wissenschaft nicht ausgeführt, während die Ziele und die methodische Vorgehensweise sehr genau beschrieben werden, da es sich bei diesem Artikel inhaltlich um eine Projektbeschreibung handelt.

Abweichungen von der klassischen Makrostruktur geistes- und sozialwissenschaftlicher Texte wie sie im von Andrea Bicsár verfassten Artikel aus der Subdisziplin Deutsch als Fremd- oder Zweitsprache auftreten (siehe Beispiel 3), werden nicht als alternative Struktur interpretiert, da die Formulierung der Fragestellung und die Wahl der Methodik in dem Artikel einer sozialwissenschaftlichen Vorgehensweise entsprechen. Hier darf also eine von der Autorin bewusst oder unbewusst vorgenommene Abweichung von der Norm angenommen werden. Solche oder ähnliche Abweichungen könnten sich eventuell auch daraus ergeben, dass AutorInnen auch von anderen herkunfts- bzw. bildungssprachlichen Kulturräumen beeinflusst sind.

Auch zeitschriftenspezifische Varianten oder Abweichungen von der klassischen Makrostruktur geistes- und sozialwissenschaftlicher Texte können nicht ausgeschlossen werden. Die Artikel von Braunmüller (2013 in Beiträge zur Geschichte der deutschen Sprache und Literatur) und Deppermann und Helmer (2013 in Zeitschrift für Sprachwissenschaft) beide im Verlag de Gruyter erschienen, verzichten z. B. gänzlich auf eine klassische Einleitung zu Gunsten eines ersten theoretischen Kapitels und eines vorangestellten Abstracts. In den Regelungen des De Gruyter Mouton journal style sheet steht darüber allerdings nichts geschrieben. Eine kurze weitere Stichprobe von 20 verschiedenen Artikeln der neuesten Jahrgänge dieses Zeitschriftenverlags hat auch tatsächlich verschiedene Realisierungsvarianten, einmal mit Einleitung und einmal ohne Einleitung, ergeben. 
Zusammenfassend wird aus dieser Pilotuntersuchung die These abgeleitet, dass - wie am Beispiel der Disziplin germanistische Linguistik demonstriert - nicht unbedingt die Disziplin sondern vielmehr die methodische Vorgehensweise, die in einer Studie angewandt wurde, um die Forschungsfrage zu bearbeiten, die Wahl der funktionalen Abschnitte beim Verfassen der Einleitung (und darüber hinaus des ganzen Artikels) bestimmt. Da die Disziplin germanistische Linguistik ein sehr breites Spektrum an wissenschaftlichen Fragestellungen und Methoden aufweist, finden sich auch verschiedene Muster sprachlicher Realisierungen. Es gibt daher vermutlich sowohl mehr Varianten innerhalb bestimmter Disziplinen als auch mehr Ähnlichkeiten zwischen verschiedenen Disziplinen als bisher in der Schreibforschung angenommen. Der Blick sollte daher in der Schreiblehre stärker dafür geschärft werden, Augenmerk auf das Formulieren der Fragestellung, die Wahl der Methode und die sich daraus ergebende Wahl der Struktur des Artikels zu legen, als sich allzu starr an Disziplingrenzen zu halten.

Um die Annahme zu überprüfen, dass die Fragestellung und die methodische Vorgehensweise die Struktur des wissenschaftlichen Artikels entscheidend mitbestimmt, wäre es natürlich empfehlenswert noch ein größeres Textkorpus zu untersuchen. In einer solchen Analyse könnten die Gemeinsamkeiten und Unterschiede zwischen verschieden methodischen Herangehensweisen an ein Thema und deren schriftliche Realisierung innerhalb verschiedener Subdisziplinen der germanistischen Linguistik noch stärker herausgearbeitet und mit anderen Disziplinen, wie z. B. der Geschichtswissenschaft, der Literaturwissenschaft oder der Naturwissenschaft verglichen werden. Das Ergebnis wäre eine Verfeinerung dieses Rasters der Makrostruktur und eine Anpassung für bestimmte methodische Vorgehensweisen, die für den Unterricht wissenschaftlichen Schreibens von unmittelbarer Relevanz wären, da es den Lehrenden und den Lernenden einerseits klar definierte Grenzen und andererseits Varianten und Alternativen innerhalb einer Disziplin aufzuzeigen vermag. Besonders hilfreich wären von dieser Untersuchung inspirierte Anleitungen für SchreibtrainerInnen, junge Forschende, die noch wenig Erfahrung im Verfassen wissenschaftlicher Artikel haben, oder für wissenschaftliche AutorInnen, die Deutsch als Zweit- oder Fremdsprache erlernen oder die in einem nicht-deutschsprachigen Sprach- und Kulturraum wissenschaftlich sozialisiert wurden.

\section{Literaturverzeichnis}

Adamzik, Kirsten/Antos, Gerd/Jakobs, Eva-Maria (eds.) (1997): Domänen- und kulturspezifisches Schreiben. Frankfurt a. M.: Lang.

Bazerman, Charles (1988): Shaping Written Knowledge. The Genre and Activity of the Experimental Article in Science. Wisconsin: University of Wisconsin Press.

Biber, Douglas et al. (2002): „Speaking and writing in the university: A multi-dimensional comparison". TESOL Quarterly 36/1: 9-48. doi: 10.2307/3588359.

Busch-Lauer, Ines-Andrea (1997): „Schreiben in der Medizin. Eine Untersuchung anhand deutscher und englischer Fachtexte“. In: Jakob, Eva-Maria/Knorr, Dagmar (eds.): Schreiben in den Wissenschaften. Frankfurt a. M., Lang: 45-61.

Busch-Lauer, Ines-Andrea (2001): Fachtexte im Kontrast: Eine linguistische Analyse zu den Kommunikationsbereichen Medizin und Linguistik. Frankfurt a. M.: Lang. 
Danneberg, Lutz (1993): „Darstellungsformen in Geistes- und Naturwissenschaften“. In: Brenner, Peter J. (ed.): Geist, Geld und Wirtschaft. Arbeits- und Darstellungsformen von Literaturwissenschaft. Frankfurt a. M., Suhrkamp: 99-137.

Ehlich, Konrad (1995): „Die Lehre der deutschen Wissenschaftssprache: sprachliche Strukturen, didaktische Desiderate“. In: Kretzenbacher, Heinz L./Weinrich, Harald (eds.): Linguistik der Wissenschaftssprache. Berlin/New York, de Gruyter: 325-351.

Ehlich, Konrad/Graefen, Gabriele (2001): „Sprachliches Handeln als Medium diskursiven Denkens. Überlegungen zur sukkursiven Einübung in die deutsche Wissenschaftskommunikation“. Jahrbuch Deutsch als Fremdsprache 27. Ismaning, Hueber: 351-378.

Ehlich, Konrad/Steets, Angelika (eds.). (2003): Wissenschaftlich schreiben - lehren und lernen. Berlin: de Gruyter.

Eggins, Susan/Martin, James (1997): "Genres and Registers of Discourse”. In: van Dijk, Teun Adrianus (ed.): Discourse as Structure and Process. Discourse Studies: A Multidisciplinary Introduction 1. London, Sage: 230-256.

Göpferich, Susanne (1995): Textsorten in Naturwissenschaft und Technik. Pragmatische Typologie - Kontrastierung - Translation. Tübingen: Narr.

Graefen, Gabriele (1997): Der Wissenschaftliche Artikel - Textart und Textorganisation. Frankfurt a. M.: Lang.

Gray, Bethany Ekle (2011): Exploring Academic Writing through Corpus Linguistics: When Discipline Tells only Part of the Story. Doktorarbeit, Northern Arizona University. http://gradworks.umi.com/34/90/3490519.html, [30.05.2016].

Gruber, Helmut/Huemer, Birgit (in Druck): „Studentisches Schreiben erforschen und lehren: Grundlagenforschung und ihre Umsetzung in ein Kursprogramm“. Zeitschrift für Hochschuldidaktik.

Gruber, Helmut (2011): „Wissenschaftliches Schreiben“. In: Haberscheid, Stephan (ed.): Textsorten und sprachliche Handlungsmuster. Linguistische Typologien der Kommunikation. Berlin: de Gruyter: 559-577.

Gruber, Helmut/Huemer, Birgit/Rheindorf, Markus (2009): Wissenschaftliches Schreiben. Ein Praxisbuch für Studierende der Geistes- und Sozialwissenschaften. Wien: Böhlau.

Gruber, Helmut et al. (2006): Genre, Habitus und wissenschaftliches Schreiben. Wien: LIT.

Halliday, Michael/Mattgiessen, Christian (2014): Introduction to Functional Grammar. $4^{\text {th }}$ Edition. Oxon: Routledge.

Halliday, Michael/Martin, James R. (1993): Writing Science. Literacy and Discursive Power. New York: Tailor/Francis.

Huemer, Birgit/Rheindorf, Markus/Gruber, Helmut (2012). Abstract, Exposé und Forschungsantrag. Eine Schreibanleitung für Studierende und junge Forschende. Wien: Böhlau.

Hyland, Ken (1998): Hedging in Scientific Research Articles. Amsterdam: Benjamins. Hyland, Ken (2009): Teaching and Researching Writing. 2nd Edition. London: Longman. Lewin, Beverly/Fine, Jonathan/Young, Lynne (2001): Expository Discourse: A Genre-based Approach to Social Science Tests. London: Continuum.

Swales, John Malcolm (1990): Genre Analysis. English in Academic and Research Settings. Michigan: Cambridge University Press. 
Swales, John Malcolm (2004): Research Genres. Explorations and Applications. Michigan: Cambridge University Press.

Swales, John Malcolm/Feak, Christine (2009): English in Today's Research World. A Writing Guide (revised and expanded edition). Michigan: University of Michigan Press.

Swales, John Malcolm/Feak, Christine (2012): Academic Writing for Graduate Students. Essential Tasks and Skills. $3^{\text {rd }}$ edition. Michigan: University of Michigan Press.

\section{Korpus}

Beißwenger, Michael/Lemnitzer, Lothar (2013): „Aufbau eines Referenzkorpus zur deutschsprachigen internetbasierten Kommunikation als Zusatzkomponente für die Korpora im Projekt, Digitales Wörterbuch der deutschen Sprache‘ (DWDS)““. Journal for Language Technology and Computational Linguistics 28/2: 1-22. (ZGL 2014 unter Korpuslinguistik.)

Bicsár, Andrea (2012): „Kribbeln im Bauch: Emotionen im Deutschen als Fremdsprache. Eine empirische Studie“. Jahrbuch der ungarischen Germanistik: 179-204. (ZGL 2014 unter Deutsch als Fremd-/Zweitsprache.)

Braunmüller, Kurt (2013): „Morphologische Komplexität des Deutschen im Vergleich mit den skandinavischen Sprachen - typologisch und sprachhistorisch“. Beiträge zur Geschichte der deutschen Sprache und Literatur: 317-341. (ZGL 2014 unter Sprachtypologie.)

Bücker, Jörg (2013): „Jein: Formen und Funktionen einer Dialogpartikel in Mündlichkeit und Schriftlichkeit". Zeitschrift für germanistische Linguistik: 189-211. (ZGL 2014 unter Grammatik.)

Deppermann, Arnulf/Helmer, Henrike (2013): „Zur Grammatik des Verstehens im Gespräch: Inferenzen anzeigen und Handlungskonsequenzen ziehen mit also und dann". Zeitschrift für Sprachwissenschaft: 1-40. (ZGL 2014 unter Gesprächslinguistik.)

Domke, Christine (2013): „Ortsgebundenheit als distinktives Merkmal in der Textanalyse“. Zeitschrift für germanistische Linguistik: 102-126. (ZGL 2014 unter Textlinguistik)

Groß, Thomas/Osborne, Timothy (2013): „Katena und Konstruktion: Ein Vorschlag zu einer dependenziellen Konstruktionsgrammatik“. Zeitschrift für Sprachwissenschaft: 41-74. (ZGL 2014 unter Syntax.)

Hartmann. Stefan (2013): „Zwischen Transparenz und Lexikalisierung: Das Wortbildungsmuster X-ung(e) im Mittelhochdeutschen“. Beiträge zur Geschichte der deutschen Sprache und Literatur: 159-183. (ZGL 2014 unter Sprachgeschichte.)

Kaltz, Barbara (2013): „Zwischen den Stühlen: Paul Lévy (1887-1962) und die französische Germanistik in der ersten Hälfte des 20. Jahrhunderts“. Historiographia linguistica: 409432. (ZGL 2014 unter Geschichte der Linguistik.)

Klein, Franz-Josef (2009): „Pinturas, figuras, letras. Zur Darstellung logographischer und phonographischer Schriftsysteme bei José de Acosta und Gregorio Garcí“. Historiographia Linguistica XXXVI:1: 1-17.

Pentzold, Christian/Fraas, Claudia/Meier, Stefan (2013): „Online-mediale Texte: Kommunikationsformen, Affordanzen, Interfaces“. Zeitschrift für germanistische Linguistik: 81-101. (ZGL 2014 unter Textlinguistik.) 
Spagnolo, Marta Lupica (2013): „Morphologische Produktivität in deutschsprachigen Texten nicht nativer Autoren. Eine korpuslinguistische Analyse“. Zeitschrift für germanistische Linguistik: 339-376. (ZGL 2014 unter Linguistik literarischer Sprache.)

Speyer, Augustin (2013): „Performative Mündlichkeitsnähe als Faktor für die Objektstellung im Mittel- und Frühneuhochdeutschen“. Beiträge zur Geschichte der deutschen Sprache und Literatur: 342-377. (ZGL 2014 unter Sprachgeschichte.)

Szatmári, Petra (2011): „Affixoide - Pro und Kontra. Jahrbuch der ungarischen Germanistik: 156-175. (ZGL 2014 unter Wortbildung.)

ZGL (Zeitschrift für germanistische Linguistik) 2014; 42/1: 152-162. 